\title{
ON THE CONVERGENCE OF MODIFIED ISHIKAWA ITERATION PROCESS TO COMMON FIXED POINTS OF TWO NEARLY LIPSCHITZIAN MAPPINGS IN HYPERBOLIC SPACES
}

\author{
Anju Panwar ${ }^{1}$, Ravi Parkash Bhokal ${ }^{2} \S$ \\ ${ }^{1,2}$ Department of Mathematics \\ M.D. University \\ Rohtak, 124001, Haryana, INDIA
}

\begin{abstract}
In this paper, we prove strong and weak convergence results of modified Ishikawa iterative process to common fixed points of two nearly lipschitzian mappings in the framework of hyperbolic spaces.Our results extend and improve results of [4] and [11] from uniformly convex Banach spaces and CAT (0) spaces to hyperbolic spaces.
\end{abstract}

AMS Subject Classification: 47H09, 47H10

Key Words: hyperbolic space, nearly lipschitzian mappings, condition (A), condition (B), opial condition

\section{Introduction}

Let $(X, d)$ be a metric space. A geodesic is a curve $\gamma: I \rightarrow R$ where $I$ is an interval such that for all $x, y \in I$,

$$
d(\gamma(x), \gamma(y))=|x-y|
$$

Thus a geodesic is any curve between two points on a surface. It plays significant

Received: March 15, 2016

Published: June 17, 2016

${ }^{\S}$ Correspondence author
(C) 2016 Academic Publications, Ltd.

url: www.acadpubl.eu 
role in the study of various geometric shapes. Hyperbolic space is an important example of the geodesic space. Hyperbolic space play key role in the study of various branches of applied mathematics and geometric group theory. Moreover fixed point theory of non-linear mappings in the set up of hyperbolic space is a fascinating field of research.Hyperbolic space is inherit rich geometrical structure. Its structural properties play a key role in obtaining new results in metric fixed point theory.Our aim is to prove strong and weak convergence results of modified Ishikawa iterative process to common fixed points of two nearly lipschitzian mappings in the framework of hyperbolic space defined by Kohlenbach [5] which is:

A metric space $(X, d)$ is a hyperbolic space if there exists a map $W: X \times$ $X \times[0,1] \rightarrow X$ satisfying:

1. $d(u, W(x, y, \alpha)) \leq \alpha d(u, x)+(1-\alpha) d(u, y)$,

2. $d(W(x, y, \alpha), W(x, y, \beta))=|\alpha-\beta| d(x, y)$,

3. $W(x, y, \alpha)=W(y, x,(1-\alpha))$,

4. $d(W(x, z, \alpha), W(y, w, \alpha)) \leq \alpha d(x, y)+(1-\alpha) d(z, w)$, for all $x, y, z, w \in X$ and $\alpha, \beta \in[0,1]$.

A subset $H$ of a hyperbolic space $X$ is convex if $W(x, y, \alpha) \in H$ for all $x, y \in H$ and $\alpha \in[0,1]$. The class of hyperbolic spaces given by Kohlenbach [5] contains all the normed linear spaces and convex subsets as well as CAT(0) spaces.

\section{Preliminaries}

To prove the our main results, we are required the following definitions, concepts and the lemmas:

Let $F(T)=\{T x=x: x \in H\}$ denotes the set of fixed points of a self map $T$ defined on $H$ and $F(t) \cap F(T)$ denotes the set of common fixed points of two mappings $t$ and $T$. We use the symbols $\rightarrow$ for the strong convergence and $\rightarrow$ for the weak convergence.

We begin with the following definitions.

Definition 1. Let $H$ be a non-empty subset of a metric space $X$. The mapping $T: H \rightarrow H$ is said to be nonexpansive if

$$
d(T x, T y) \leq d(x, y) \text { for all } x, y \in H
$$


Definition 2. Let $H$ be a non-empty subset of a metric space $X$. The mapping $T: H \rightarrow H$ is said to be uniformly $L$-Lipschitzian if for each $n \in N$, there exists a positive integer $L>0$ such that

$$
d\left(T^{n} x, T^{n} y\right) \leq L d(x, y) \text { for all } x, y \in K
$$

The class of nearly Lipschitzian mappings was introduced by Sahu [10] and it is an important generalization of the class of Lipschitzian mappings.

Definition 3. Let $H$ be a non-empty subset of a metric space $X$ and fix a sequence $\left\{a_{n}\right\}$ in $[0, \infty)$ with $a_{n} \rightarrow 0$. A mapping $T: H \rightarrow H$ is said to be nearly Lipschitzian with respect to $\left\{a_{n}\right\}$ if for each $n \in N$, there exists a constant $k_{n} \geq 0$ such that

$$
d\left(T^{n} x, T^{n} y\right) \leq k_{n}\left(d(x, y)+a_{n}\right) \text { for all } x, y \in H
$$

The infimum of the constants $k_{n}$ for which (2.1) holds is denoted by $\eta\left(T^{n}\right)$, called the nearly Lipschitz constant of the mapping $T$. A nearly Lipschitzian mapping $T$ with the sequence $\left\{\left(a_{n}, \eta\left(T^{n}\right)\right\}\right.$ is said to be:

1. nearly nonexpansive if $\eta\left(T^{n}\right)=1$ for all $n \in N$;

2. nearly uniformly $k$-Lipschitzian if $\eta\left(T^{n}\right) \leq k$ for all $n \in N$.

The case of two mappings in iterative procedures is under study since Das and Debate [1] and recently the convergence of the iterative procedure to the common fixed points of two mappings has been studied by many authors including $[4,11,12]$.

In 1974, Ishikawa [3] introduced famous two step iterative procedure for one mapping and recently Hafiz Fukhar-ud-din [2] modified the Ishikawa iterative procedure to the case of two mappings.

Definition 4. Let $H$ be a non-empty subset of a hyperbolic space $X$ and $T$ and $t$ be two self mappings on $H$.For $x_{1} \in H$ consider the sequence $\left\{x_{n}\right\}$ defined by

$$
\begin{aligned}
x_{n+1} & =W\left(T^{n} y_{n}, t^{n} x_{n}, \alpha_{n}\right), \\
y_{n} & =W\left(T^{n} x_{n}, t^{n} x_{n}, \beta_{n}\right), \quad n \geq 1,
\end{aligned}
$$

where $0<\alpha_{n}<1$ and $0 \leq \beta_{n}<1$ is called the modified Ishikawa iterative procedure. 
Definition 5. [9] A mapping $t: H \rightarrow H$ with $F(t) \neq \phi$ in $H$ is said to satisfy condition $(A)$ with respect to the sequence $\left\{x_{n}\right\}$ if there is a nondecreasing function $\rho:[0,1) \rightarrow[0,1)$ with $\rho(0)=0$ and $\rho(r)>0$ for all $r \in(0, \infty)$ such that for all $p \in F(t)$, we have

$$
\rho\left(d\left(x_{n}, p\right)\right) \leq d\left(x_{n}, \rho\left(x_{n}\right)\right) \text { for all } n \geq 1
$$

Definition 6. [11] Two mappings $t, T: H \rightarrow H$, where $H$ is a non-empty subset of a metric space $(X, d)$, are said to satisfy condition $(B)$ if there exists a non-decreasing function $\rho:[0, \infty) \rightarrow[0, \infty)$ with $\rho(0)=0$ and $\rho(r)>0$ for all $r \in(0, \infty)$ such that

$$
\alpha d(x, t x)+\beta d(x, T x) \geq \rho(d(x, p))
$$

for all $x \in H$ and $p \in F(T) \cap F(t)$, where

$$
d(x, p)=\inf \{d(x, p): p \in F(T) \cap F(t) \neq \phi\}
$$

and $\alpha$ and $\beta$ are two non-negative real numbers such that $\alpha+\beta=1$.

Opial [7] condition plays an important role in the study of weak convergence.

Definition 7. A hyperbolic space $X$ is said to satisfy Opial condition if for any sequence $\left\{x_{n}\right\}$ in $X$ such that $\left\{x_{n}\right\} \rightarrow x$ implies that

$$
\limsup _{n \rightarrow \infty} d\left(x_{n}, x\right)<\limsup _{n \rightarrow \infty} d\left(x_{n}, y\right),
$$

for all $y \in X$ with $x \neq y$.

Examples of the spaces satisfying Opial condition are Hilbert spaces and all $\ell^{p}(1<p<\infty)$ spaces.

Definition 8. A mapping $T: X \rightarrow X$ is said to be demiclosed at zero, if for any sequence $\left\{x_{n}\right\}$ in $X$, the condition $\left\{x_{n}\right\} \rightarrow x$ and $T\left(x_{n}\right) \rightarrow 0$ imply $T x=0$.

In the sequel the following lemmas will be needed.

Lemma 9. (see [6]) Let $(X, d, W)$ be a uniformly convex hyperbolic space with monotone modulus of uniform convexity $\eta$. Let $x \in X$ and $\left\{\alpha_{n}\right\}$ be a sequence in $[a, b]$ for some $a, b \in(0,1)$.If $\left\{x_{n}\right\}$ and $\left\{y_{n}\right\}$ are sequences in $X$ such that

$$
\limsup _{n \rightarrow \infty} d\left(x_{n}, x\right) \leq r
$$




$$
\begin{array}{r}
\limsup _{n \rightarrow \infty} d\left(y_{n}, x\right) \leq r, \\
\lim _{n \rightarrow \infty} d\left(W\left(x_{n}, y_{n}, \alpha_{n}\right), x\right)=r,
\end{array}
$$

for some $r \geq 0$, then

$$
\lim _{n \rightarrow \infty} d\left(x_{n}, y_{n}\right)=0 .
$$

Lemma 10. (see [8]) Let $\left\{\alpha_{n}\right\},\left\{\beta_{n}\right\}$ and $\left\{\delta_{n}\right\}$ be sequences of nonnegative real numbers such that

$$
\alpha_{n+1} \leq\left(1+\delta_{n}\right) \alpha_{n}+\beta_{n}, \text { for all } n \geq 1
$$

If $\sum_{n=1}^{\infty} \delta_{n}<\infty$ and $\sum_{n=1}^{\infty} \beta_{n}<\infty$, then $\lim _{n \rightarrow \infty} \alpha_{n}$ exists.

\section{Main Results}

Lemma 11. Let $H$ be a non-empty compact convex subset of a hyperbolic space $X$ and let $t, T: H \rightarrow H$ be two nearly lipschitzian mappings with sequences $\left\{a_{n}^{\prime}, \eta_{1}\left(t^{n}\right)\right\}$ and $\left\{a_{n}^{\prime \prime}, \eta_{2}\left(T^{n}\right)\right\}$ respectively such that $\Sigma_{n=1}^{\infty} a_{n}^{\prime}<\infty$, $\Sigma_{n=1}^{\infty} a_{n}^{\prime \prime}<\infty$ and $F(t) \bigcap F(T) \neq \phi$. Let $\left\{x_{n}\right\}$ be a sequence of modified Ishikawa iteration defined by (2.2). Then

$$
\lim _{n \rightarrow \infty} d\left(x_{n}, p\right) \text { exists for all } p \in F(t) \bigcap F(T) \text {. }
$$

Proof. Let $p \in F(t) \cap F(T)$ and W.L.O.G. we may take $\eta_{1}\left(t^{n}\right) \leq \eta_{2}\left(T^{n}\right)$. From (2.2) we have

$$
\begin{aligned}
d\left(y_{n}, p\right) & =d\left(W\left(T^{n} x_{n}, t^{n} x_{n}, \beta_{n}\right), p\right) \\
& \leq\left(1-\beta_{n}\right) d\left(T^{n} x_{n}, p\right)+\beta_{n} d\left(t^{n} x_{n}, p\right) \\
& \leq\left(1-\beta_{n}\right)\left[\eta_{2}\left(T^{n}\right)\left(d\left(x_{n}, p\right)+a_{n}^{\prime \prime}\right)\right]+\beta_{n}\left[\eta_{1}\left(t^{n}\right)\left(d\left(x_{n}, p\right)+a_{n}^{\prime}\right)\right] .
\end{aligned}
$$

Let $a_{n}=\max \left\{a_{n}^{\prime}, a_{n}^{\prime \prime}\right\}$. Then

$$
\begin{aligned}
d\left(y_{n}, p\right) \leq & \left(1-\beta_{n}\right)\left[\eta_{2}\left(T^{n}\right)\left(d\left(x_{n}, p\right)+a_{n}\right)\right]+\beta_{n}\left[\eta_{1}\left(t^{n}\right)\left(d\left(x_{n}, p\right)+a_{n}\right)\right] \\
\leq & \left(1-\beta_{n}\right) \eta_{2}\left(T^{n}\right) d\left(x_{n}, p\right)+\left(1-\beta_{n}\right) \eta_{2}\left(T^{n}\right) a_{n} \\
& +\beta_{n} \eta_{1}\left(t^{n}\right) d\left(x_{n}, p\right)+\beta_{n} \eta_{1}\left(t^{n}\right) a_{n} \\
\leq & {\left[\left(1-\beta_{n}\right) \eta_{2}\left(T^{n}\right)+\beta_{n} \eta_{1}\left(t^{n}\right)\right] d\left(x_{n}, p\right) } \\
& +\left[\left(1-\beta_{n}\right) \eta_{2}\left(T^{n}\right)+\beta_{n} \eta_{1}\left(t^{n}\right)\right] a_{n}
\end{aligned}
$$




$$
\begin{aligned}
& \leq \eta_{1}\left(t^{n}\right) \eta_{2}\left(T^{n}\right)\left[\left(1-\beta_{n}\right) d\left(x_{n}, p\right)+\beta_{n} d\left(x_{n}, p\right)\right]+\eta_{2}\left(T^{n}\right) a_{n} \\
& \leq \eta_{1}\left(t^{n}\right) \eta_{2}\left(T^{n}\right) d\left(x_{n}, p\right)+\eta_{2}\left(T^{n}\right) a_{n} .
\end{aligned}
$$

Again using (3.1) and (2.2), we get

$$
\begin{aligned}
d\left(x_{n+1}, p\right)= & d\left(W\left(T^{n} y_{n}, t^{n} x_{n}, \alpha_{n}\right), p\right) \\
\leq & \left(1-\alpha_{n}\right) d\left(T^{n} y_{n}, p\right)+\alpha_{n} d\left(t^{n} x_{n}, p\right) \\
\leq & \left(1-\alpha_{n}\right)\left[\eta_{2}\left(T^{n}\right)\left(d\left(y_{n}, p\right)+a_{n}^{\prime \prime}\right)\right]+\alpha_{n}\left[\eta_{1}\left(t^{n}\right)\left(d\left(x_{n}, p\right)+a_{n}^{\prime}\right)\right] \\
\leq & \left(1-\alpha_{n}\right) \eta_{2}\left(T^{n}\right) d\left(y_{n}, p\right)+\alpha_{n} \eta_{1}\left(t^{n}\right) d\left(x_{n}, p\right) \\
& +\left[\left(1-\alpha_{n}\right) \eta_{2}\left(T^{n}\right)+\alpha_{n} \eta_{1}\left(t^{n}\right)\right] a_{n} \\
\leq & \left(1-\alpha_{n}\right) \eta_{2}\left(T^{n}\right)\left[\eta_{1}\left(t^{n}\right) \eta_{2}\left(T^{n}\right) d\left(x_{n}, p\right)+\eta_{2}(T) a_{n}\right] \\
& +\alpha_{n} \eta_{1}\left(t^{n}\right) d\left(x_{n}, p\right)+\left[\left(1-\alpha_{n}\right) \eta_{2}\left(T^{n}\right)+\alpha_{n} \eta_{1}\left(t^{n}\right)\right] a_{n} \\
\leq & \left(1-\alpha_{n}\right) \eta_{1}\left(t^{n}\right) \eta_{2}\left(T^{n}\right)^{2} d\left(x_{n}, p\right)+\left(1-\alpha_{n}\right) \eta_{2}\left(T^{n}\right)^{2} a_{n} \\
& +\alpha_{n} \eta_{1}\left(t^{n}\right) d\left(x_{n}, p\right)+\eta_{2}\left(T^{n}\right) a_{n} \\
\leq & {\left[\left(1-\alpha_{n}\right) \eta_{1}\left(t^{n}\right) \eta_{2}\left(T^{n}\right)^{2}+\alpha_{n} \eta_{1}\left(t^{n}\right)\right] d\left(x_{n}, p\right) } \\
& +\left[\left(1-\alpha_{n}\right) \eta_{2}\left(T^{n}\right)^{2}+\eta_{2}\left(T^{n}\right)\right] a_{n}
\end{aligned}
$$

Let

$$
\omega_{n}=\left[\left(1-\alpha_{n}\right) \eta_{1}\left(t^{n}\right) \eta_{2}\left(T^{n}\right)^{2}+\alpha_{n} \eta_{1}\left(t^{n}\right)-1\right],
$$

and

$$
\vartheta_{n}=\left[\left(1-\alpha_{n}\right) \eta_{2}\left(T^{n}\right)^{2}+\eta_{2}\left(T^{n}\right)\right] a_{n} .
$$

Therefore we have

$$
d\left(x_{n+1}, p\right) \leq\left(1+\omega_{n}\right) d\left(x_{n}, p\right)+\vartheta_{n},
$$

Then by the given conditions we conclude that $\sum_{n=1}^{\infty} \omega_{n}<\infty$ and $\sum_{n=1}^{\infty} \vartheta_{n}<$ $\infty$. Using Lemma 10 and (3.2) we have $\lim _{n \rightarrow \infty} d\left(x_{n}, p\right)$ exists.

Lemma 12. Let $H$ be a non-empty compact convex subset of a hyperbolic space $X$ and let $t, T: H \rightarrow H$ be two uniformly continuous and nearly lipschitzian mappings with sequences $\left\{a_{n}^{\prime}, \eta_{1}\left(t^{n}\right)\right\}$ and $\left\{a_{n}^{\prime \prime}, \eta_{2}\left(T^{n}\right)\right\}$ respectively such that $\Sigma_{n=1}^{\infty} a_{n}^{\prime}<\infty, \Sigma_{n=1}^{\infty} a_{n}^{\prime \prime}<\infty$ and $F(t) \bigcap F(T) \neq \phi$. Let $\left\{x_{n}\right\}$ be a sequence of modified Ishikawa iteration defined by $(2.2)$. If $d\left(x, T^{n} x\right) \leq$ $d\left(t^{n} x, T^{n} x\right)$ and $d\left(x, t^{n} x\right) \leq d\left(T^{n} x, t^{n} x\right)$ for all $x \in H$.

Then

$$
\lim _{n \rightarrow \infty} d\left(x_{n}, t x_{n}\right)=0
$$


and

$$
\lim _{n \rightarrow \infty} d\left(x_{n}, T x_{n}\right)=0 .
$$

Proof. From Lemma 11 we have $\lim _{n \rightarrow \infty} d\left(x_{n}, p\right)$ exists.

Suppose that $\lim _{n \rightarrow \infty} d\left(x_{n}, p\right)=\xi$. Since $d\left(t^{n} x_{n}, p\right) \leq \eta_{1}\left(t^{n}\right)\left(d\left(x_{n}, p\right)+a_{n}^{\prime}\right)$ implies that

$$
\limsup _{n \rightarrow \infty} d\left(t^{n} x_{n}, p\right) \leq \xi .
$$

Also $d\left(T^{n} x_{n}, p\right) \leq \eta_{2}\left(T^{n}\right)\left(d\left(x_{n}, p\right)+a_{n}^{\prime \prime}\right)$ implies that

$$
\limsup _{n \rightarrow \infty} d\left(T^{n} x_{n}, p\right) \leq \xi
$$

Also Lemma 11 yields that

$$
\limsup _{n \rightarrow \infty} d\left(y_{n}, p\right) \leq \xi
$$

Hence

$$
\limsup _{n \rightarrow \infty} d\left(T^{n} y_{n}, p\right) \leq \limsup _{n \rightarrow \infty} \eta_{2}\left(T^{n}\right)\left(d\left(y_{n}, p\right)+a_{n}^{\prime \prime}\right) \leq \xi .
$$

Since

$$
\begin{aligned}
\xi & =\lim _{n \rightarrow \infty} d\left(x_{n+1}, p\right) \\
& =\lim _{n \rightarrow \infty} d\left(W\left(T^{n} y_{n}, t^{n} x_{n}, \alpha_{n}\right), p\right) .
\end{aligned}
$$

From Lemma 9, (3.3), (3.6) and (3.7) we conclude that

$$
\lim _{n \rightarrow \infty} d\left(T^{n} y_{n}, t^{n} x_{n}\right)=0 .
$$

Now

$$
\begin{aligned}
d\left(x_{n+1}, T^{n} y_{n}\right) & =d\left(W\left(T^{n} y_{n}, t^{n} x_{n}, \alpha_{n}\right), T^{n} y_{n}\right) \\
& \leq \alpha_{n} d\left(T^{n} y_{n}, t^{n} x_{n}\right) .
\end{aligned}
$$

Therefore

$$
d\left(x_{n+1}, T^{n} y_{n}\right) \rightarrow 0 \text { as } n \rightarrow \infty .
$$

Also

$$
\begin{aligned}
d\left(x_{n+1}, t^{n} x_{n}\right) & =d\left(W\left(T^{n} y_{n}, t^{n} x_{n}, \alpha_{n}\right) t^{n} x_{n}\right) \\
& \leq\left(1-\alpha_{n}\right) d\left(T^{n} y_{n}, t^{n} x_{n}\right) .
\end{aligned}
$$


Hence

$$
d\left(x_{n+1}, t^{n} x_{n}\right) \rightarrow 0 \text { as } n \rightarrow \infty .
$$

Now

$$
\begin{aligned}
d\left(x_{n+1}, p\right) & \leq d\left(x_{n+1}, T^{n} y_{n}\right)+d\left(T^{n} y_{n}, p\right) \\
& \leq d\left(x_{n+1}, T^{n} y_{n}\right)+\eta_{2}\left(T^{n}\right)\left(d\left(y_{n}, p\right)+a_{n}^{\prime \prime}\right)
\end{aligned}
$$

implies that

$$
\xi \leq \liminf _{n \rightarrow \infty} d\left(y_{n}, p\right) .
$$

By (3.5) and (3.10) we have

$$
\lim _{n \rightarrow \infty} d\left(y_{n}, p\right)=\xi
$$

Thus

$$
\lim _{n \rightarrow \infty} d\left(W\left(T^{n} x_{n}, t^{n} x_{n}, \beta_{n}\right), p\right)=\xi .
$$

Therefore we have from $(3.3),(3.4),(3.11)$ and Lemma 9

$$
\lim _{n \rightarrow \infty} d\left(T^{n} x_{n}, t^{n} x_{n}\right)=0 .
$$

Now

$$
\begin{aligned}
d\left(x_{n}, t^{n} x_{n}\right) & \leq d\left(x_{n}, T^{n} x_{n}\right)+d\left(T^{n} x_{n}, t^{n} x_{n}\right) \\
& \leq d\left(t^{n} x_{n}, T^{n} x_{n}\right)+d\left(T^{n} x_{n}, t^{n} x_{n}\right) \\
& \leq 2 d\left(t^{n} x_{n}, T^{n} x_{n}\right) \rightarrow 0 .
\end{aligned}
$$

Again

$$
\begin{aligned}
d\left(x_{n}, T^{n} x_{n}\right) & \leq d\left(x_{n}, t^{n} x_{n}\right)+d\left(t^{n} x_{n}, T^{n} x_{n}\right) \\
& \leq d\left(T^{n} x_{n}, t^{n} x_{n}\right)+d\left(t^{n} x_{n}, T^{n} x_{n}\right) \\
& \leq 2 d\left(t^{n} x_{n}, T^{n} x_{n}\right) \rightarrow 0 .
\end{aligned}
$$

By the uniform continuity of $T, \lim _{n \rightarrow \infty} d\left(x_{n}, T^{n} x_{n}\right)=0$ implies that

$$
\lim _{n \rightarrow \infty} d\left(T x_{n}, T^{n+1} x_{n}\right)=0 .
$$

Also

$$
\begin{aligned}
d\left(x_{n+1}, x_{n}\right) & \leq d\left(W\left(T^{n} y_{n}, t^{n} x_{n}, \alpha_{n}\right), x_{n}\right) \\
& \leq\left(1-\alpha_{n}\right) d\left(T^{n} y_{n}, x_{n}\right)+\alpha_{n} d\left(t^{n} x_{n}, x_{n}\right)
\end{aligned}
$$




$$
\begin{aligned}
& \leq\left(1-\alpha_{n}\right) d\left(T^{n} y_{n}, x_{n+1}\right)+\left(1-\alpha_{n}\right) d\left(x_{n+1}, x_{n}\right) \\
& +\alpha_{n} d\left(t^{n} x_{n}, x_{n}\right) .
\end{aligned}
$$

This yields that

$$
\alpha_{n} d\left(x_{n+1}, x_{n}\right) \leq\left(1-\alpha_{n}\right) d\left(T^{n} y_{n}, x_{n+1}\right)+\alpha_{n} d\left(t^{n} x_{n}, x_{n}\right) .
$$

Using (3.8), (3.12) and (3.15) we have

$$
\lim _{n \rightarrow \infty} d\left(x_{n+1}, x_{n}\right)=0 .
$$

Also

$$
\begin{aligned}
d\left(x_{n}, T x_{n}\right) & \leq d\left(x_{n}, x_{n+1}\right)+d\left(x_{n+1}, T^{n+1} x_{n+1}\right) \\
& +d\left(T^{n+1} x_{n+1}, T^{n+1} x_{n}\right)+d\left(T^{n+1} x_{n}, T x_{n}\right) \\
& \leq d\left(x_{n}, x_{n+1}\right)+d\left(x_{n+1}, T^{n+1} x_{n+1}\right)+\eta_{2}\left(T^{n}\right) d\left(x_{n+1}, x_{n}\right) \\
& +d\left(T^{n+1} x_{n}, T x_{n}\right) \\
& \leq\left(1+\eta_{2}\left(T^{n}\right)\right) d\left(x_{n+1}, x_{n}\right)+d\left(x_{n+1}, T^{n+1} x_{n+1}\right) \\
& +d\left(T^{n+1} x_{n}, T x_{n}\right) .
\end{aligned}
$$

Using (3.13), (3.14) and (3.16) in the above inequality we obtain,

$$
\lim _{n \rightarrow \infty} d\left(x_{n}, T x_{n}\right)=0 .
$$

By using similar arguments we can show that $\lim _{n \rightarrow \infty} d\left(x_{n}, t x_{n}\right)=0$. This completes the proof.

Theorem 13. Let $H$ be a non-empty compact convex subset of a hyperbolic space $X$ and let $t, T: H \rightarrow H$ be two nearly lipschitzian mappings with sequences $\left\{a_{n}^{\prime}, \eta_{1}\left(t^{n}\right)\right\}$ and $\left\{a_{n}^{\prime \prime}, \eta_{2}\left(T^{n}\right)\right\}$ respectively such that $\Sigma_{n=1}^{\infty} a_{n}^{\prime}<\infty$, $\sum_{n=1}^{\infty} a_{n}^{\prime \prime}<\infty$ and $F(t) \bigcap F(T) \neq \phi$. Let $\left\{x_{n}\right\}$ be a sequence of modified Ishikawa iteration defined by (2.2) and also suppose that the mappings $t$ and $T$ satisfy condition $(A)$. Then $\left\{x_{n}\right\}$ converges strongly to a common fixed point of $t$ and $T$.

Proof. From Lemma 12, we have $\lim _{n \rightarrow \infty} d\left(T x_{n}, x_{n}\right)=0$. Since $T$ satisfies condition $(A)$, we have $\lim _{n \rightarrow \infty} d\left(T x_{n}, p\right)=0$ for all $p \in F(t) \cap F(T)$. Hence there exists a sequence $\left\{\alpha_{n_{i}}\right\}$ of $\left\{x_{n}\right\}$ and $\nu_{k} \subset F(T)$ such that for all $k$ we have $d\left(\alpha_{n_{i}}, \nu_{k}\right)<\frac{\epsilon}{2}$. Now we claim that $\left\{\nu_{k}\right\}$ is a Cauchy sequence in $H$. Note that

$$
d\left(\nu_{k+1}, \nu_{k}\right) \leq d\left(\nu_{k+1}, \alpha_{n_{k+1}}\right)+d\left(\alpha_{n_{k+1}}, \nu_{k}\right)
$$




$$
\leq \frac{\epsilon}{2}+\frac{\epsilon}{2}=\epsilon
$$

This shows that $\left\{\nu_{k}\right\}$ is a Cauchy sequence in $H$. Now completeness of $H$ implies that there must exist some $y \in H$ such that $\left\{\nu_{k}\right\} \rightarrow y$. Also $d\left(\nu_{k}, T y\right)=$ $d\left(T \nu_{k}, T y\right) \leq \eta_{2}\left(T^{n}\right)\left(d\left(\nu_{k}, y\right)+a_{n}^{\prime \prime}\right) \rightarrow 0$ as $k, n \rightarrow \infty$. Thus we conclude that $\lim _{k \rightarrow \infty} d\left(\nu_{k}, T y\right)=d(y, T y)=0$. Hence $d(y, T y)=0$ implies that $y \in F(T)$. Since $H$ is compact so there exists a subsequence $\left\{x_{n_{i}}\right\}$ of $\left\{x_{n}\right\}$ such that $\left\{x_{n_{i}}\right\} \rightarrow y$. Again from Lemma 12, we have

$$
\lim _{n \rightarrow \infty} d\left(t x_{n_{i}}, x_{n_{i}}\right)=0
$$

Now

$$
\begin{aligned}
d(y, t y) & \leq d\left(y, x_{n_{i}}\right)+d\left(x_{n_{i}}, t x_{n_{i}}\right)+d\left(t x_{n_{i}}, t y\right) \\
& \leq d\left(y, x_{n_{i}}\right)+d\left(x_{n_{i}}, t x_{n_{i}}\right)+\eta_{1}\left(t^{n}\right)\left(d\left(x_{n_{i}}, y\right)+a_{n}^{\prime}\right) \\
& \rightarrow 0
\end{aligned}
$$

It follows that $d(y, t y)=0$ implies that $y \in F(t)$. Hence $y$ is the common fixed point of the mappings $t$ and $T$. Now we prove that $\left\{x_{n}\right\}$ converges strongly to $y$. We have $\lim _{n_{i} \rightarrow \infty} d\left(x_{n_{i}}, y\right)=0$. Also Lemma 11 asserts that $\lim _{n \rightarrow \infty} d\left(x_{n}, y\right)$ exists. The only possible case is $\lim _{n \rightarrow \infty} d\left(x_{n}, y\right)=\lim _{n_{i} \rightarrow \infty} d\left(x_{n_{i}}, y\right)=0$. Hence $\left\{x_{n}\right\}$ converges strongly to a common fixed point of $t$ and $T$.

Theorem 14. Let $H$ be a non-empty closed convex subset of a complete hyperbolic space $X$ and let $t, T: H \rightarrow H$ be two nearly lipschitzian mappings with sequences $\left\{a_{n}^{\prime}, \eta_{1}\left(t^{n}\right)\right\}$ and $\left\{a_{n}^{\prime \prime}, \eta_{2}\left(T^{n}\right)\right\}$ respectively such that $\Sigma_{n=1}^{\infty} a_{n}^{\prime}<\infty, \Sigma_{n=1}^{\infty} a_{n}^{\prime \prime}<\infty$ and $F(t) \bigcap F(T) \neq \phi$. Let $\left\{x_{n}\right\}$ be a sequence of modified Ishikawa iteration defined by (2.2). If either $\liminf _{n \rightarrow \infty} d\left(x_{n}, p\right)=0$ or $\limsup _{n \rightarrow \infty} d\left(x_{n}, p\right)=0$, for all $p \in F(t) \cap F(T)$. Then the sequence $\left\{x_{n}\right\}$ converges strongly to common fixed point of $t$ and $T$.

Proof. From Lemma 11, we have $\lim _{n \rightarrow \infty} d\left(x_{n}, p\right)$ exists. Using given assumptions $\liminf _{n \rightarrow \infty} d\left(x_{n}, p\right)=0$ or $\limsup _{n \rightarrow \infty} d\left(x_{n}, p\right)=0$,for all $p \in F(t) \cap F(T)$, we have $\lim _{n \rightarrow \infty} d\left(x_{n}, p\right)=0$.

Now we claim that $\left\{x_{n}\right\}$ is a Cauchy sequence in $H$. Using the similar arguments as that of [12, Theorem 3.1], we conclude $\left\{x_{n}\right\}$ is a Cauchy sequence in $H$. Since $X$ is complete so $\left\{x_{n}\right\}$ is convergent. Assume that $\lim _{n \rightarrow \infty} x_{n}=\alpha$. Also $H$ is closed, therefore $\alpha \in H$. Now we claim that $\alpha$ is a common fixed point 
of $t$ and $T$. From Lemma 12, $\lim _{n \rightarrow \infty} d\left(x_{n}, T x_{n}\right)=0$ implies that $d(\alpha, T \alpha)=0$ therefore $\alpha \in F(T)$. Similarly it can be proved that $\alpha \in F(t)$. Hence $\alpha \in$ $F(t) \cap F(T)$. This completes the proof.

Corollary 15. Let $H$ be a non-empty closed convex subset of a complete hyperbolic space $X$ and let $t, T: H \rightarrow H$ be two

1. nearly non-expansive,

2. nearly uniformly $k$-Lipschitzian

mappings with the sequences $\left\{a_{n}^{\prime}, \eta_{1}\left(t^{n}\right)\right\}$ and $\left\{a_{n}^{\prime \prime}, \eta_{2}\left(T^{n}\right)\right\}$ respectively satisfying all the conditions of the theorem (14).Let $\left\{x_{n}\right\}$ be a sequence of modified Ishikawa iteration defined by (2.2). Then the sequence $\left\{x_{n}\right\}$ converges strongly to a common fixed point of $t$ and $T$.

Proof. 1. Taking $\eta_{1}\left(t^{n}\right)=\eta_{2}\left(T^{n}\right)=1$ in the theorem (14).

2. Taking $\eta_{1}\left(t^{n}\right), \eta_{2}\left(T^{n}\right) \leq k$ in the theorem (14).

Theorem 16. Let $H$ be a non-empty closed convex subset of a complete hyperbolic space $X$ and let $t, T: H \rightarrow H$ be two nearly lipschitzian mappings with sequences $\left\{a_{n}^{\prime}, \eta_{1}\left(t^{n}\right)\right\}$ and $\left\{a_{n}^{\prime \prime}, \eta_{2}\left(T^{n}\right)\right\}$ respectively such that $\Sigma_{n=1}^{\infty} a_{n}^{\prime}<$ $\infty, \Sigma_{n=1}^{\infty} a_{n}^{\prime \prime}<\infty$ and $F(t) \bigcap F(T) \neq \phi$. Let $\left\{x_{n}\right\}$ be a sequence of modified Ishikawa iteration defined by (2.2). If either $t$ or $T$ is semi-compact then the sequence $\left\{x_{n}\right\}$ converges strongly to a common fixed point of $t$ and $T$.

Proof. Suppose $T$ is semi-compact. Then there exists a subsequence $\left\{\mu_{n_{j}}\right\}$ of $\left\{x_{n}\right\}$ such that such that $\mu_{n_{j}} \rightarrow p \in H$. From Lemma $12 \lim _{n \rightarrow \infty} d\left(x_{n}, T x_{n}\right)=0$ implies that

$$
\lim _{n_{j} \rightarrow \infty} d\left(\mu_{n_{j}}, T \mu_{n_{j}}\right)=0
$$

Hence we have

$$
\begin{aligned}
d(p, T p) & \leq d\left(p, \mu_{n_{j}}\right)+d\left(\mu_{n_{j}}, T \mu_{n_{j}}\right)+d\left(T \mu_{j}, T p\right) \\
& \leq d\left(p, \mu_{n_{j}}\right)+d\left(\mu_{n_{j}}, T \mu_{n_{j}}\right)+\eta_{2}\left(T^{n}\right)\left(d\left(p, \mu_{n_{j}}\right)+a_{n}^{\prime \prime}\right) \\
& \leq\left(1+\eta_{2}\left(T^{n}\right)\right) d\left(p, \mu_{n_{j}}\right)+d\left(\mu_{n_{j}}, T \mu_{n_{j}}\right)+\eta_{2}\left(T^{n}\right) a_{n}^{\prime \prime} \rightarrow 0 .
\end{aligned}
$$

Thus $p \in F(T)$. Similarly it can be proved that $p \in F(t)$. Hence $p \in F(t) \cap$ $F(T)$.

Now we claim that the sequence $\left\{x_{n}\right\}$ converges strongly to $p$. From Lemma 11 we have $\lim _{n \rightarrow \infty} d\left(x_{n}, p\right)$ exists. As $\mu_{n_{j}} \rightarrow p \in F(t) \cap F(T)$ implies that $x_{n} \rightarrow$ 
$p \in F(t) \cap F(T)$. This proves that $\left\{x_{n}\right\}$ converges strongly to a common fixed point of $t$ and $T$.

Now we prove following strong convergence theorem using condition $(B)$, which is an application of the Theorem (16).

Theorem 17. Let $H$ be a non-empty closed convex subset of a complete hyperbolic space $X$ and let $t, T: H \rightarrow H$ be two nearly lipschitzian mappings with sequences $\left\{a_{n}^{\prime}, \eta_{1}\left(t^{n}\right)\right\}$ and $\left\{a_{n}^{\prime \prime}, \eta_{2}\left(T^{n}\right)\right\}$ respectively such that $\Sigma_{n=1}^{\infty} a_{n}^{\prime}<$ $\infty, \sum_{n=1}^{\infty} a_{n}^{\prime \prime}<\infty$ and $F(t) \bigcap F(T) \neq \phi$. Let $\left\{x_{n}\right\}$ be a sequence of modified Ishikawa iteration defined by (2.2). Let $t$ and $T$ satisfy condition $(B)$, then the sequence $\left\{x_{n}\right\}$ converges strongly to a common fixed point of $t$ and $T$.

Proof. From the condition $(B)$ and Lemma 12 we have for some $p \in F(t) \cap$ $F(T)$

$$
\begin{aligned}
\lim _{n \rightarrow \infty} \rho\left(d\left(x_{n}, p\right)\right) & \leq \alpha \lim _{n \rightarrow \infty} d\left(x_{n}, t x_{n}\right)+\beta \lim _{n \rightarrow \infty} d\left(x_{n}, T x_{n}\right) \\
& =0 .
\end{aligned}
$$

where $\rho:[0, \infty) \rightarrow[0, \infty)$ is a non-decreasing function such that $\rho(0)=0$ and $\rho(t)>0$ for all $t \in[0, \infty)$. Hence $\lim _{n \rightarrow \infty} d\left(x_{n}, p\right)=0$. Now all the hypothesis of the theorem (16) are satisfied. Hence we have $\left\{x_{n}\right\}$ converges strongly to a common fixed point of $t$ and $T$.

At last we give our weak convergence result, In which $I: X \rightarrow X$ denotes the identity mapping.

Theorem 18. Let $H$ be a non-empty closed convex subset of a complete hyperbolic space $X$ satisfing Opial condition. Let $t, T: H \rightarrow H$ be two nearly lipschitzian mappings with sequences $\left\{a_{n}^{\prime}, \eta_{1}\left(t^{n}\right)\right\}$ and $\left\{a_{n}^{\prime \prime}, \eta_{2}\left(T^{n}\right)\right\}$ respectively such that $\Sigma_{n=1}^{\infty} a_{n}^{\prime}<\infty, \Sigma_{n=1}^{\infty} a_{n}^{\prime \prime}<\infty$ and $F(t) \cap F(T) \neq \phi$. Let $\left\{x_{n}\right\}$ be a sequence of modified Ishikawa iteration defined by (2.2). If mappings $(I-t)$ and $(I-T)$ are demiclosed at zero, then the sequence $\left\{x_{n}\right\}$ converges weakly to a common fixed point of $t$ and $T$.

Proof. Let $p \in F(t) \bigcap F(T)$. Now by Lemma 11, $\lim _{n \rightarrow \infty} d\left(x_{n}, p\right)$ exists. Since $X$ is uniformly convex therefore $H$ is weakly compact. Then there exists a subsequence $\left\{\alpha_{n_{k}}\right\} \subset\left\{x_{n}\right\}$ such that $\left\{\alpha_{n_{k}}\right\}$ converges weakly to some $\rho \in H$. Also the mappings $(I-t)$ and $(I-T)$ are demiclosed at zero, then by definition $(I-t) \rho=0$ and $(I-T) \rho=0$ implies that $\rho \in F(t) \bigcap F(T)$.

Now we shall prove that $\left\{x_{n}\right\}$ converges weakly to $\rho \in F(t) \bigcap F(T)$. On the contrary, let us suppose that there exists a subsequence $\left\{\beta_{n_{j}}\right\}$ of $\left\{x_{n}\right\}$ such 
that $\left\{\beta_{n_{j}}\right\}$ converges weakly to $\rho_{1} \in H$ such that $\rho \neq \rho_{1}$. Again using the assumption that mappings $(I-t)$ and $(I-T)$ are demiclosed at zero, we have $(I-t) \rho_{1}=0$ and $(I-T) \rho_{1}=0$ implies that

$$
\rho_{1} \in F(t) \bigcap F(T)
$$

Therefore by Lemma 11, $\lim _{n \rightarrow \infty} d\left(x_{n}, \rho_{1}\right)$ exists. Using Opial condition we have

$$
\limsup _{n \rightarrow \infty} d\left(x_{n}, \rho\right)<\limsup _{n \rightarrow \infty} d\left(x_{n}, \rho_{1}\right)
$$

and

$$
\limsup _{n \rightarrow \infty} d\left(x_{n}, \rho_{1}\right)<\limsup _{n \rightarrow \infty} d\left(x_{n}, \rho\right) .
$$

By using (3.17), (3.18) and Lemma 11 we conclude that

$$
\lim _{n \rightarrow \infty} d\left(x_{n}, \rho\right)=\lim _{n \rightarrow \infty} d\left(x_{n}, \rho_{1}\right) \text {. }
$$

which is a contradiction so $\rho=\rho_{1}$. Therefore $\left\{x_{n}\right\}$ converges weakly to a common fixed point of $t$ and $T$.

\section{References}

[1] G. Das, J.P. Debate, Fixed points of quasi-nonexpansive mappings, Indian Journal of Pure and Applied Mathematics, 17 (1986), 1263-1269.

[2] H. Fukhar-ud-din, Convergence of Ishikawa type iteration process for three quasinonexpansive mappings in a convex metric space, Verista, 23, No. 2 (2015), 83-92.

[3] S. Ishikawa, Fixed points by a new iteration methods, Proc. Amer. Math. Soc., 44 (1974), 147-150.

[4] P.K. Jhade, R.A. Rashwan, A.S. Saluja, On the convergence of Ishikawa iterative process for a pair of single-valued and multi-valued nonexpansive mappings, Gulf Journal of Mathematics, 4, No. 2 (2014), 68-77.

[5] U. Kohlenbach, Some logical metatheorems with applications in functional analysis, Trans. Amer. Math. Soc., 357, No. 1 (2005), 89-128.

[6] A.R. Khan, H. Fukhar-ud-din, M.A.A. Khan, An implicit algorithm for two finite families of nonexpansive maps in hyperbolic spaces, Fixed Point Theory and Applications (2012), $1-12$.

[7] Z. Opial, Weak convergence of the sequence of successive approximations for nonexpansive mappings, Bull. Amer.Math. Soc., 73 (1967), 591-597.

[8] L. Qihou, Iterative sequences for asymptotically quasi-nonexpansive mappings with error members, J. Math. Anal. Appl., 259 (2001), 18-24.

[9] H.F. Senter, W.G. Dotson, Approximating fixed points of nonexpansive mappings, Proc. Amer. Math. Soc., 44 (1974), 375-380. 
[10] D.R. Sahu, Fixed points of demi-continuous nearly Lipschitzian mappings in Banach spaces, Comment Math. Univ. Carol., 46 (2005), 653-666.

[11] G.S. Saluja, Convergence of modified S-iteration process for two generalized asymptotically quasi-nonexpansive mappings in CAT(0) spaces, Mathematica Moravica, 19, No. 1 (2015), 19-31.

[12] G.S. Saluja, Strong and $\triangle$-Convergence of modified two step iterations for nearly asymptotically nonexpansive mappings in hyperbolic spaces, International Journal of Analysis and Applications, 8, No. 1 (2015), 39-52. 